\title{
Essai d'explication et de quantification des morphologies fluviales à partir de la théorie du régime
}

\author{
M. Ramette \\ Ingénieur Conseil \\ 1 , rue des Ruisseaux \\ 92140 Clamart
}

La configuration la plus simple d'une morphologie fluviale est le lit unique et rectiligne; les caractéristiques géométriques moyennes d'un tel lit : largeur, profondeur et pente, ont fait l'objet de nombreuses études expérimentales pour servir de support à la réalisation de canaux artificiels, de géométrie simple et restant, autant que possible, stables dans le temps.

Les études entreprises par LACEY pour définir les caractéristiques de canaux d'irrigation et publiées en 1947 sous le titre "A general theory of flow in alluvium channels ", sont restées parmi les plus connues, sous le nom de "théorie du régime".

L'objet du présent «essai » est de montrer que les configurations morphologiques les plus courantes des cours d'eau naturels : méandres, lits en tresses (braided beds) correspondent probablement à une "dégénérescence " du lit rectiligne obéissant à la théorie du régime, dégénérescence liée à une diminution relative du transport solide.

Les relations morphologiques découlant des hypothèses qui seront exposées sont comparées à des relations expérimentales, en particulier à celles établies récemment au cours des études de la Jamuna dans sa traversée du Bangladesh (Brahmapoutre dans la zone indienne).

Tentative to explain and to quantify the fluvial morphological processes from the Regime theory

The most elementary feature of a river's morphology is a straight single bed. The mean geometrical characteristics of such a bed: depth, width, slope, have been the subject of numerous experimental studies to support the design of man-made stable channels.

Mainly for irrigation purpose, LACEY has developed a "general theory of flow in alluvium channels " (1930-1947) which is known as "Regime theory".

The objective of the present article is to show that most of morphological features: single meandering beds, straight and meandering braided beds, are probably arising from an initial single straight bed under the "regime theory" laws, when the solid discharge is growing gradually less.

The theoretical relations deriving from the hereafter exposed hypothesis are compared with the ones compiled from experimental results and especially from the Jamuna river field measurements in Bangladesh. 


\section{Notations}

\section{Grandeurs dimensionnelles}

$Q$ : débit liquide $\left(\mathrm{m}^{3} / \mathrm{s}\right)$

$P$ : périmètre mouillé $(\mathrm{m})$

$A$ : section mouillée $\left(\mathrm{m}^{2}\right)$

$R:$ rayon hydraulique $=A / P(\mathrm{~m})$

$H$ : profondeur moyenne $(\mathrm{m})$

$B$ : largeur moyenne $(\mathrm{m})$

$d$ : diamètre moyen du sédiment $(\mathrm{m})$

$k$ : coefficient de rugosité de Manning-Strickler $\left(\mathrm{m}^{1 / 3} / \mathrm{s}\right)$

$G$ : débit solide $\left(\mathrm{m}^{3 / \mathrm{s}}\right)$

$\rho$ : rayon de courbure des méandres $(\mathrm{m})$

$\lambda: 1 / 2$ longueur d'onde des méandres $(\mathrm{m})$

$\gamma: 1 / 2$ amplitude des méandres (m)

\section{Grandeurs sans dimensions}

$S$ : pente de la ligne d'énergie $(\mathrm{m} / \mathrm{m})$

a : densité du sédiment (supposée égale à 2,6)

$\beta \quad$ : allongement $=B / H$

$\phi \quad$ : coefficient de friction tangentielle : $R S /(\partial-1) d$

\section{Formules utilisées}

Coefficient de rugosité - fonds plats

$$
k=k_{r}=\frac{21}{d^{1 / 6}}\left(\frac{d}{H}\right)^{1 / 24} .
$$

Coefficient de rugosité - dunes (avec

$$
\begin{gathered}
\left.\partial=2,6 \text { et } k_{s}<k_{r}\right) \\
k=k_{s}=1,3 k_{r}\left(\frac{0,06+0,41 \phi^{2}}{\phi}\right)^{5 / 8} .
\end{gathered}
$$

Formule de MANNING-STRICKLER pour l'écoulement

$$
Q=k A R^{2 / 3} S^{1 / 2}
$$

ou, en introduisant les grandeurs sans dimensions et invariants :

Fonds plats $\left(k=k_{r}\right)$

$$
\begin{aligned}
X_{0} & =Q S^{17 / 8} / d^{5 / 2} \\
& =72,1 \phi^{21 / 8}\left(\frac{\beta+2}{\beta}\right)^{47 / 24} \cdot \beta .
\end{aligned}
$$

Dunes $\left(k=k_{s}\right)$

$$
\begin{aligned}
X_{1} & =Q S^{17 / 8} / d^{5 / 2} \\
& =91,4 \phi^{2}\left(0,06+0,41 \phi^{2}\right)^{5 / 8}\left(\frac{\beta+2}{\beta}\right)^{47 / 24} \cdot \beta .
\end{aligned}
$$

Nota : On trouvera dans les documents réf. (a) et réf. $(b)$ une justification des formules utilisées.
Formules de transport solide, dans le domaine charriage et suspension à partir de la formule d'ENGELUND-HANSEN (E. H.) (4.0)

Formule d'E. H. dans le domaine fonds plats $\left(k=k_{r}\right)$

$$
\begin{aligned}
Y_{01} & =G S^{5 / 4} / d^{5 / 2} \\
& =25,1 \phi^{15 / 4}\left(\frac{\beta+2}{\beta}\right)^{11 / 12} \cdot \beta .
\end{aligned}
$$

Formule d'E. H. dans le domaine dunes $\left(k=k_{s}\right)$

$$
\begin{aligned}
Y_{02} & =G S^{5 / 4} / d^{5 / 2} \\
& =4,03 \phi^{5 / 2}\left(0,06+0,41 \phi^{2}\right)^{5 / 4}\left(\frac{\beta+2}{\beta}\right)^{11 / 12} \cdot \beta .
\end{aligned}
$$

Formules de transport solide, dans le domaine charriage seul, à partir de la formule de MEYER-PETER (M. P.) : (4.1)

Formule de M. P. dans le domaine fonds plats $\left(k=k_{r}\right)$

$$
\begin{aligned}
Y_{11} & =G S / d^{5 / 2} \\
& =72 \phi(\phi-0,047)^{3 / 2}(\beta+2) .
\end{aligned}
$$

Formule de M.P. dans le domaine dunes $\left(k=k_{s}\right)$

$$
\begin{aligned}
Y_{12}=G S / d^{5 / 2}=72 \phi & {\left[1,426\left(\frac{0,06+0,41 \phi^{2}}{\phi}\right)^{15 / 16} \times\right.} \\
& \times \phi-0,047]^{3 / 2}(\beta+2) .
\end{aligned}
$$




\section{A. LES ÉQUATIONS DU « RÉGIME »}

Les équations de LACEY concernent le périmètre mouillé et le rayon hydraulique de canaux stables et rectilignes ; exprimées en unités S. I., elles s'écrivent comme suit :

$$
\begin{aligned}
& P=4.81 Q^{12} \\
& R=0.127 Q^{1 / 3} / d^{1 / 6} .
\end{aligned}
$$

La validité de ces équations peut, a priori, paraître suspecte du fait de leur simplicité et de l'absence du paramètre pente $(S)$. Cependant, comme elles rendent compte avec un excellent coefficient de corrélation d'un très grand nombre d'observations, il est évident qu'elles doivent pouvoir être rattachées aux équations classiques de l'hydraulique.

\section{A.1. Analogie avec les équations classiques de l'écoulement}

La formule (3): $Q=k A R^{2 / 3} S^{1 / 2}$ peut être transformée, en remarquant que, par définition $R=A / P$, soit

$$
Q=k P R^{5 / 3} S^{12} \text {. }
$$

Il existe une infinité de solutions permettant de calculer le couple $P, R$ pour $Q, k$ et $S$ donnés. La solution est unique si l'on se donne une relation $f(P, R)$.

Nous faisons l'hypothèse que cette relation est fixée par les équations de LACEY, soit :

$$
P / R=38 Q^{1 / 6} d^{1 / 6} .
$$

Dans ces conditions, on peut écrire :

$$
Q=k .38 R^{83} Q^{1 / 6} d^{16} S^{1 / 2}
$$

\section{A.1.0. Hypothèse de l'écoulement sur fonds plats}

Dans un écoulement sur fonds plats,

$$
k=k_{r}=\frac{21}{d^{1 / 6}}\left(\frac{d}{H}\right)^{1 / 24} .
$$

L'introduction de cette valeur avec $H \# R$ dans l'équation précédente permet d'exprimer $R$ :

$$
R=0,0784 Q^{0.317} /\left(S^{0.19} \cdot d^{0.016}\right)
$$

On en déduit :

$$
P=2,98 Q^{0.484} d^{0.151} / S^{0.19}
$$

\section{A.1.1. Hypothèse de l'écoulement sur dunes}

L'équation (3.1) de l'écoulement en présence de dunes, peut être simplifiée en remarquant d'une part que $\phi^{2}\left(0,06+0,41 \phi^{2}\right)^{5 / 8} \# 0,64 \phi^{3.035}$ dans une large plage de variation de $\phi(>0,4)$, d'autre part que

$$
\left(\frac{\beta+2}{\beta}\right)^{47 / 24} \cdot \beta \#\left(\frac{\beta+2}{\beta}\right)^{2} \cdot \beta=P / R .
$$

Dans ces conditions, avec $P / R=38 Q^{1 / 6} d^{1 / 6}$, l'équation (3.1) s'écrit :

$$
\frac{Q S^{178}}{d^{5 / 2}} \# 91,4\left(0,64 \phi^{3.035}\right) 38 Q^{1 / 6} d^{1 / 6} .
$$

Sachant que $\phi=R \cdot S / 1,6 d$, on en déduit :

$$
\begin{aligned}
& R=0,126 Q^{0.275} d^{0.121} / S^{0.3} \\
& P=4,8 Q^{0.411} d^{0.288} / S^{0.3} .
\end{aligned}
$$

\section{A.2. Conclusions préliminaires}

Si l'on en juge uniquement par les exposants de $Q$ dans les expressions de $P$ et $R$ on constate que les équations de LACEY s'accordent davantage avec les équations théoriques de l'écoulement sur fonds plats qu'avec celles sur dunes.

L'identification des coefficients des équations (I) et (II) avec respectivement ceux des équations (IV) et (V) conduit à admettre que dans tous les canaux « stables " étudiés par LACEY, la pente et le diamètre du sédiment étaient liés par la relation suivante :

$$
d^{0.151} / S^{0.19}=1,61 Q^{0,016} .
$$

Remarquons que le bien-fondé de cette dernière relation repose en particulier sur la validité de l'expression. donnant la valeur du coefficient de rugosité $k=k_{r}$ sur fonds plats :

$$
k=\frac{21}{d^{1 / 6}}\left(\frac{d}{H}\right)^{1 / 24} .
$$


Cette relation expérimentale se vérifie assez bien dans une large gamme de variation de $d$ et $H$, mais pour un allongement $\beta$ suffisamment grand pour que l'influence des parois du canal soit négligeable. Lorsque $\beta$ est relativement faible : inférieur à 40 ou 50 , ce qui est le cas pour beaucoup de canaux étudiés par LACEY, la rugosité réelle peut être plus importante que la rugosité théorique et donc le coefficient de rugosité $k$ plus faible.

Si par exemple on estime $k=0,8 k_{r}$, la relation entre $S$ et $d$ serait la suivante :

$$
d^{0.151} / S^{0.19}=1,48 Q^{0,016} \text {. }
$$

Les relations (VIII) ou (IX) pourraient exprimer la transition entre fonds plats et dunes (cf. A.3.2.1). Leur validité, sur le plan expérimental est discutée aux chapitres B.4.1.2 et C.

En définitive: De ce qui précède on peut tirer les conclusions préliminaires suivantes:

1. Les canaux en « régime" sont par définition des canaux rectilignes ;

2. Les équations du " régime " traduisent assez correctement un écoulement sur fond plat ; elles permettent d'exprimer l'allongement $\beta$ à partir de la relation

$$
\left(\frac{\beta+2}{\beta}\right)^{2} \cdot \beta=38 Q^{1 / 6} d^{1 / 6} \text {. }
$$

3. Pour que les équations de LACEY soient compatibles avec les équations théoriques de l'écoulement sur fonds plats, $S$ et $d$ doivent être liés par une relation du type (VIII) pour les fortes valeurs de l'allongement $\beta$ ou (IX) pour les faibles valeurs de $\beta$.

Ecoulement rectiligne et fonds sensiblement plats seraient donc deux conditions nécessaires pour que le lit soit stable.

- Ces conditions paraissent, a priori, physiquement justifiées :

- un lit à méandres par le jeu des érosions dépôts "glisse " vers l'aval et ne peut donc être considéré comme stable, même si sa configuration reste identique à elle-même dans sa translation;

- l'apparition de dunes sur le fond est un facteur d'instabilité directionnelle de l'écoulement, surtout si ces dunes sont alternées, ce qui est le cas lorsque l'allongement dépasse 15 à 20 (réf. $c$ ).

Si les deux conditions énoncées paraissent nécessaires, elles ne sont probablement pas suffisantes pour assurer la stabilité du lit.

Dans le chapitre suivant, on essaye de dégager une signification physique complémentaire aux équations de LACEY, liée au transport solide.

\section{A.3. Représentation graphique des équations du régime}

Dans ce qui suit, on se place dans l'hypothèse d'une rugosité minimale du lit ; on admet donc que l'équation (VIII) est respectée :

$$
d^{0,151} / S^{0,19}=1,61 Q^{0,016} .
$$

\section{A.3.1. Equations de l'écoulement}

Les équations générales théoriques de l'écoulement sur fonds plats (avec rugosité minimale) sont représentées dans les axes $X_{0}, \phi$, avec $\beta$ comme paramètre, sur la figure 1 .

$$
\begin{aligned}
X_{0} & =Q S^{17 / 1 / 8} / d^{5 / 2} \\
& =72,1 \phi^{21 / 8}\left(\frac{\beta+2}{\beta}\right)^{47 / 24} \cdot \beta .
\end{aligned}
$$

\section{A.3.2. Equations du régime}

Les équations du régime imposent deux conditions complémentaires, l'une liée au fait que $\beta$ n'est pas une variable indépendante :

$$
\left(\frac{\beta+2}{\beta}\right)^{2} \cdot \beta=38 Q^{1 / 6} d^{1 / 6}
$$

l'autre, que la pente est liée à la dimension du sédiment par la relation (VIII).

Ces deux conditions introduites dans l'équation (3.0) se traduisent par la relation suivante:

$$
X_{0} \# 8.10^{4} \phi^{3.29} d^{0.415} \text {. }
$$

La relation (X) est représentée sur la figure 1 dans les axes $X_{0}, \phi$ avec $d$ comme paramètre dans la plage $0,110^{-3}<d<1 \mathrm{~m}$.

\section{A.3.2.1. Interprétation de l'équation (X)}

Tout point $X_{0}, \phi, d$ correspond à un écoulement en « régime ". Le problème se pose de la signification d'un point représentatif d'un écoulement, $X_{0}, \phi$, situé hors de la courbe iso $d$ correspondant au régime.

Soit $A$, le point représentatif d'un écoulement en " régime " de débit $Q$ de pente $S_{A}$, avec $d_{A}=0,510^{-3} \mathrm{~m}$ (fig. 1), $X_{0 A}, \phi_{A}$ les coordonnées correspondantes.

Le point $B$ tel que $X_{0 B}=X_{0 A}$ et $d_{A}=0,510^{-3}$, à gauche de la courbe iso $d=0,510^{-3}$, peut parfaitement correspondre à un écoulement sur fonds plats permettant d'évacuer le débit $Q$ sous la même pente $S_{B}=S_{A}$; il peut aussi correspondre à un écoulement de débit $Q$ sur fonds plats, mais avec $d_{B}=510^{-3} \mathrm{~m}$ (fig. 1) et $S_{B}>S_{A}$. En effet, $X_{0 A}=X_{0 B}$ implique

$$
\frac{Q S_{A}^{17 / 8}}{d_{A}^{5 / 2}}=\frac{Q S_{B}^{17 / 8}}{d_{B}^{5 / 2}} \quad ; d_{B}>d_{A} \text { donc } S_{B}>S_{A} \text {. }
$$

Le point $B$ peut donc être figuratif d'un écoulement plus rugueux que celui correspondant au régime. 


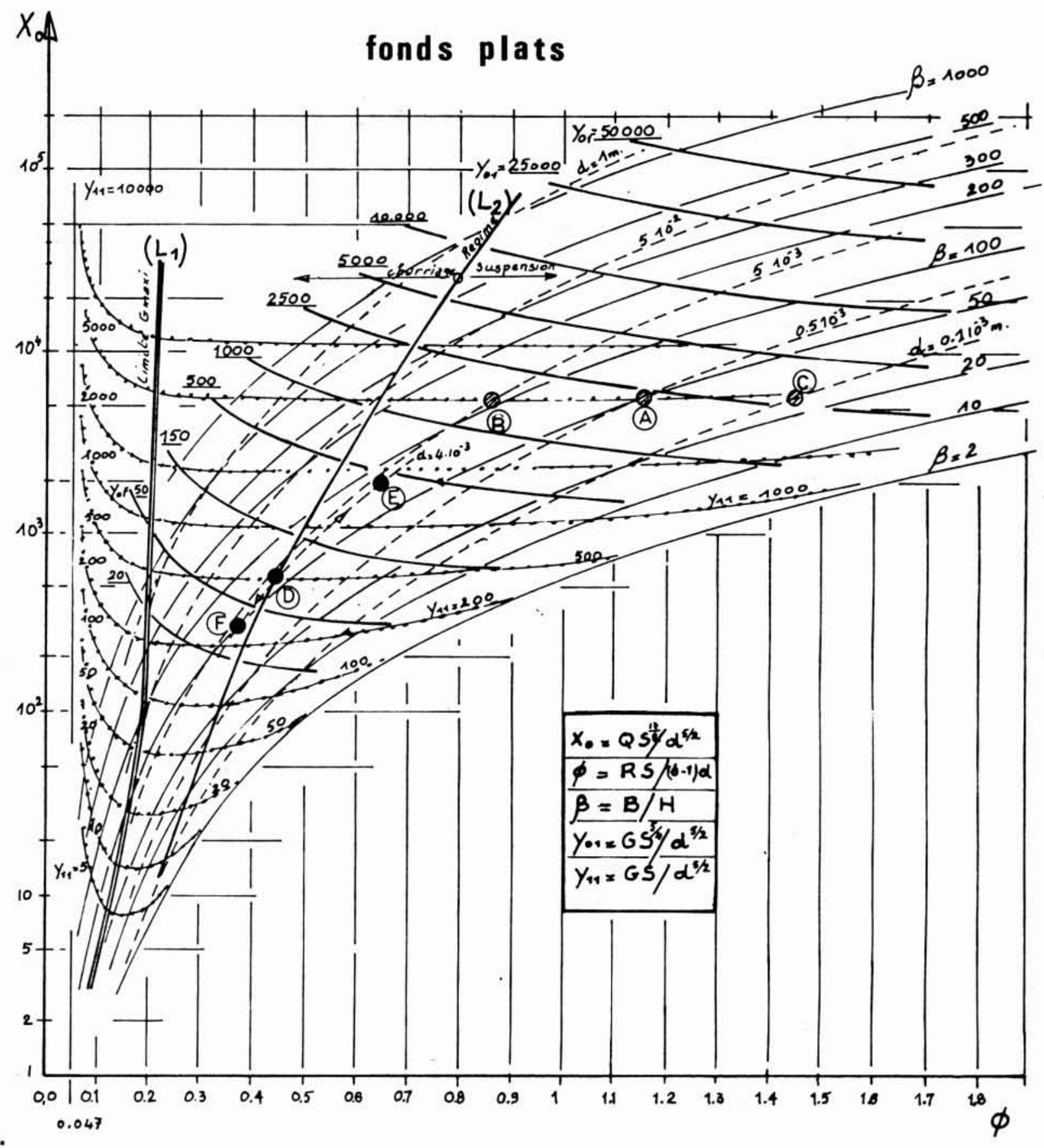

Un raisonnement identique peut être tenu pour un point représentatif $C$ tel que $X_{0 C}=X_{0 A}$, situé à droite de la courbe iso $d=0,510^{-3} \mathrm{~m}$, et qui lui aussi peut correspondre à un écoulement sur fond plat évacuant le débit $Q$ sous la pente $S_{C}=S_{A}$.

Par contre ce point représentatif ne peut correspondre à un écoulement plus rugueux que celui du régime, ni à un écoulement beaucoup plus lisse car, dans ce cas, la rugosité correspondrait à celle provoquée par un sédiment $d_{C}=0,110^{-3} \mathrm{~m}<d_{A}=0,510^{-3}$ (fig. 1).
En conclusion nous admettrons que les courbes iso $d$ séparent, à leur droite les écoulements sur fonds plats, à leur gauche des écoulements sur fonds rugueux pouvant correspondre à l'apparition des dunes.

Remarquons en effet que l'augmentation de rugosité ne correspond pas obligatoirement à l'apparition de dunes, mais peut être engendrée par une rugosité complémentaire de forme lorsque l'allongement $\beta$ est faible (cf. chapitre A.2, équation (IX)).

En outre il existe probablement une plage de transition et non pas une courbe unique iso $d$, à l'intérieur de laquelle les fonds peuvent encore être considérés comme plats. 


\section{A.3.3. Equations du transport solide sur fonds plats}

Deux types de transport solide sont considérés :

- charriage,

- charriage et suspension.

Il existe de nombreuses formules, mais celles de MEYER-PETER-Múller pour le charriage et d'EngELUND-HANSEN pour le charriage et suspension nous paraissent, d'expérience, parmi les plus fiables.

Ces deux formules présentées au chapitre «Notations et formules utilisées " et correspondant aux fonds plats sont les suivantes :

Charriage et suspension (E. H.)

$$
\begin{aligned}
Y_{01} & =G S^{5 / 4} / d^{5 / 2} \\
& =25,1 \phi^{15 / 4}\left(\frac{\beta+2}{\beta}\right)^{11 / 12} \cdot \beta .
\end{aligned}
$$

\section{Charriage (M. P.)}

$$
\begin{aligned}
Y_{11} & =G S / d^{5 / 2} \\
& =72 \phi(\phi-0,047)^{3 / 2}(\beta+2)
\end{aligned}
$$

Les relations 4.0 .1 et 4.1 .1 sont représentées sur la figure 1 dans les axes $X_{0}, \phi$ avec respectivement $Y_{01}$ et $Y_{11}$ comme paramètres.

\section{On notera les points suivants :}

A.3.3.1. Les familles de courbes $Y_{01}$ et $Y_{11}=$ constantes ont une concavité tournée vers les valeurs croissantes de $X_{0}$. Pour des valeurs de $Q, S, d$, données, c'est-à-dire pour une valeur donnée de $X_{0}$, les caractéristiques du lit permettant d'évacuer le maximum de débit solide $G$ sont déterminées par le point représentatif $X_{0}, \phi_{0}, \beta$ coïncidant avec le minimum d'une courbe $Y_{01}=$ cste s'il s'agit de charriage et suspension, ou d'une courbe $Y_{11}=$ cste s'il s'agit de charriage seul. Les valeurs de $Y_{01}$, ou de $Y_{11}$ permettent, bien entendu, de calculer le débit solide maximum $G$ correspondant.

A.3.3.2. Le lieu des minima des courbes $Y_{01}=$ cste (charriage et suspension) se situe pratiquement entre les courbes iso $\beta 10$ et 20 .

Par contre, le lieu des minima des courbes $Y_{11}=$ cste (charriage) intéresse une très large plage que l'allure des courbes permet de situer entre $\beta \# 20$ pour les fortes valeurs de $\phi$ et une limite vraisemblable (courbe $L_{1}$ ) représentée sur la figure 1 vers les plus faibles valeurs de $\phi$ comprises entre 0,1 et 0,25 .

A.3.3.3. La plupart des courbes à $d$ constant, représentées par l'équation $(\mathbf{X})$ traduisant le régime, se situent à l'intérieur des limites où l'on peut considérer que le charriage est maximum. Pour les faibles valeurs de $d=0,1$ à $0,210^{-3} \mathrm{~m}$, le débit solide en suspension est, de plus, proche de sa valeur maximale.

Pour les fortes valeurs de $d$ et pour $\phi<0,20$, les courbes iso $d$ ne correspondent plus tout à fait au charriage maximum. L'allure des courbes suggère cependant que pour $d>510^{-3} \mathrm{~m}$ elles pourraient être asymp- totiques à la courbe limite $\left(L_{1}\right)$ représentée sur la figure 1. Dans cette hypothèse qui restreint très faiblement le domaine de validité de l'équation $(\mathbf{X})$, l'ensemble des courbes (iso $d$ ) correspondrait à un débit solide maximum.

\section{A.3.3.4. Conclusions concernant le transport solide}

Parmi l'infinité de canaux permettant d'écouler un débit liquide $Q$, sous une pente $S$, avec un fond plat constitué de sédiments de dimension moyenne $d$, seuls les canaux obéissant à des équations proches de celles du régime présentent la particularité d'évacuer le maximum de débit solide ; la part du débit solide qui s'effectue par charriage est de plus maximale.

Cette particularité nous paraît être une condition complémentaire de stabilité du lit ; en effet toute modification d'une configuration initiale, par exemple par érosion du fond ou des berges, sous-entend que les matériaux issus de cette érosion peuvent être évacués par le courant sous la forme d'un transport solide qui s'ajoute donc au transport initial; si ce dernier est maximum on voit mal comment le processus de modification des fonds ou des berges pourrait s'amorcer et se développer.

Une exception cependant est à considérer, lorsque le lit, même saturé en débit solide, présente des méandres ; une érosion dans les zones concaves peut en effet être compensée par un dépôt de volume équivalent dans les zones convexes, sans que le transport solide général ne soit modifié. Ce phénomène bien connu engendre une translation des méandres mais on ne peut dans ce cas parler de stabilité du lit.

Les conditions de stabilité énoncées en A.4 débouchent donc sur la constatation qu'elles traduisent un débit solide maximum, et il faut même préciser maximum maximorum dans le cas d'un cours d'eau naturel, pour tenir compte du fait que la pente d'un lit rectiligne est maximale car elle correspond à celle de la plaine dans laquelle il s'écoule, alors que, toutes choses égales par ailleurs, un lit à méandres dans cette même plaine présente une pente moindre et a donc une capacité de transport solide plus faible.

\section{A.3.3.5. Remarques concernant la pente}

Un cours d'eau naturel ajuste sa morphologie pour évacuer, d'une part un débit liquide $Q$, d'autre part un débit solide $G$ qui lui sont imposés ; il y a toutes raisons de penser que cette morphologie se créera avec une dépense minimale d'énergie comme il en est ainsi pour la plupart des phénomènes naturels. Or, c'est précisément une caractéristique d'un lit obéissant aux lois du régime, puisque, dans ce cas, débit liquide et débit solide s'écoulent sous une pente $S$ minimale (cf. A.3.3.1).

L'hypothèse qui est faite et qui sera développée dans les chapitres suivants est que la pente de la plaine alluvionnaire correspond à la pente initiale générée par le cours d'eau, dans les conditions du régime, pour évacuer, d'une part le débit liquide $Q$ (ou une suite de débits $Q$ variables dans le temps), d'autre part, le débit solide $G$ (dont l'intégration dans le temps correspond au volume solide mis à disposition par érosion du bassin). On vérifiera aisément, à partir des relations (3.0) et 
(4.0.1), que pour $Q$ et $G$ donnés, ainsi que pour une dimension $d$ du sédiment, dans le cas d'un transport solide par charriage et suspension, la pente $S$ d'un cours d'eau " en régime " est donnée par la relation approchée suivante :

$$
S \# 12,4 G^{0.56} d^{0.64} / Q^{0.76} \text {. }
$$

En introduisant la relation (VIII) (qui reste à justifier sur le plan expérimental), on trouve une relation indépendante de $d$ :

$$
S \# 6,810^{9} G^{2.80} / Q^{3.47} .
$$

(XIbis)

Dans le cas d'un transport solide par charriage seul, la combinaison des équations (VIII), (3.0) et (4.0.1), ou encore simplement l'exploitation de la figure 1, permettent d'établir la relation approchée suivante :

$X_{0} \# 1,3 Y_{11}^{0,98}$ soit, en partant des définitions de $X_{0}$ et $Y_{11}$ :

$$
S \# 1,28 G^{0.85} d^{0.04} / Q^{0.87} \text {. }
$$

Là aussi, on constate que la pente est pratiquement indépendante de $d$.

\section{A.3.3.6. Domaines d'application des relations de MEYER-PETER et d'ENGELUND-HANSEN dans le domaine fonds plats}

Il est évidemment important de connaître par quel mode le transport solide s'effectue : charriage seul ou charriage et suspension?

A défaut d'expérimentation systématique dans ce domaine, du moins peut-on déterminer les conditions correspondant à un même transport solide $G$, calculé à partir des formules de charriage de MEYER-PETER pour le charriage d'une part, et d'ENGELUND-HANSEN d'autre part, lorsque la suspension apparaît.

La combinaison des équations générales (4.0.1) et (4.1.1) avec $G$ et $d$ constants et en remarquant que

$$
\left(\frac{\beta+2}{\beta}\right)^{11 / 12} \cdot \beta \# \beta+2,
$$

donne la condition suivante, valable pour tous les écoulements :

$$
S \# 1,4810^{-2} \phi^{11} /(\phi-0,047)^{6}
$$

Cette relation générale, associée aux équations du régime, permet de dresser le tableau ci-dessous pour différentes valeurs de $S$.

La relation $X_{0}, \phi$, notée $\left(L_{2}\right)$ est tracée sur la figure 1 . Elle peut être interprétée comme suit :

Pour tout couple de valeurs $X_{0}, \phi$, correspondant à une valeur de $d$ (par exemple $X_{0}=566, \phi=0,444$, $d=410^{-3}$ — point $D$ de la figure 1 ) le débit solide, sur fond plat par charriage et suspension est égal au débit solide sur fond plat par charriage, à condition que la pente corresponde à celle du tableau, c'est-à-dire $510^{-4}$ dans l'exemple cité. Si la pente est supérieure, le débit solide donné par la formule d'ENGELUND-HANSEN excède celui donné par la formule de MEYER-PETER (point représentatif $E$ sur la courbe $d=410^{-3}$ ).

Si la pente est inférieure c'est au contraire la formule de Meyer-Peter qui donne une valeur de transport solide plus importante (point représentatif $F$ sur la figure 1).

Nous faisons l'hypothèse que la courbe $\left(L_{2}\right)$ tracée sur la figure 1 correspond à la limite des domaines de validité de l'une et l'autre des formules lorsqu'elles sont appliquées à des écoulements obéissant aux lois du régime :

- charriage à gauche de la courbe,

- charriage et suspension à droite de la courbe.

Nota 1: Le tableau $A$ montre que si la dimension moyenne du sédiment est de $1,210^{-2} \mathrm{~m}$, le débit nécessaire pour qu'apparaisse la suspension est de $64500 \mathrm{~m}^{3} / \mathrm{s}$ et la pente au minimum de $S=10^{-3}$.

Sur la base de l'hypothèse formulée précédemment, on peut donc admettre que pour $d>1,5 \mathrm{~cm}$ environ, le transport solide s'effectue toujours par charriage, à moins d'envisager des valeurs énormes pour le débit $Q$.

Nota 2: D'après ce qui précède, $d=1,210^{-2} \mathrm{~m}$ constituerait pratiquement la borne supérieure de la dimension du sédiment pour que la suspension puisse apparaître. Par ailleurs, on peut raisonnablement penser qu'une valeur $d=0,110^{-3} \mathrm{~m}$ constitue une limite inférieure de la dimension moyenne des sédiments qui sont rencontrés dans la quasi-totalité des cours d'eau. A ces deux valeurs extrêmes de $d$, le tableau $A$ montre qu'il y correspond les valeurs de $S$ et $\phi$ suivantes pour les cours d'eau obéissant aux lois du régime :

$$
\begin{aligned}
& 510^{-5}<S<10^{-3} \\
& 0,25<\phi<0,52 .
\end{aligned}
$$

Tableau A

\begin{tabular}{|c|c|c|c|c|c|c|}
\hline$S$ & $510^{-5}$ & $10^{-4}$ & $510^{-4}$ & $10^{-3}$ & $510^{-3}$ & $10^{-2}$ \\
\hline$\phi$ & 0,25 & 0,3 & 0,444 & 0,52 & 0,744 & 0,865 \\
\hline$X_{0}$ & 17,8 & 52,8 & 566 & 1524 & 14420 & 37579 \\
\hline$Q \mathrm{~m}^{3} / \mathrm{s}$ & 2,3 & 26,7 & 6350 & 64500 & $1310^{6}$ & - \\
\hline$d \mathrm{~m}$ & $0,110^{-3}$ & $0,310^{-3}$ & $410^{-3}$ & $1,210^{-2}$ & $6,710^{-2}$ & $2110^{-2}$ \\
\hline
\end{tabular}


Nous verrons, au chapitre B.3.2 suivant, que ce domaine de variation de $\phi$ correspond à la zone de maximum de rugosité lorsque les dunes apparaissent ( $k / k$, minimum) ainsi qu'au maximum de cambrure des dunes. Dans une précédente étude (réf. $a$ ) l'hypothèse a été émise que ce maximum de cambrure des dunes pourrait correspondre à un «déferlement " provoquant l'apparition de la suspension, ce qui justifierait la frontière entre les deux types de transport solide que constitue la courbe limite $\left(L_{2}\right)$.

\section{A.4. Conclusions générales sur le régime et les caractéristiques générales du transport solide qui lui sont associées}

Les cours d'eau et canaux obéissant aux équations du régime présentent les caractéristiques suivantes :
- lit rectiligne et pente maximale, s'il s'agit de cours d'eau naturels,

- allongement $\beta$ fonction de $Q$ et $d$,

- débit solide maximum,

- fonds plats,

- les courbes iso $d$ (ou tout au moins une frange autour de ces courbes) constituent la limite entre fonds plats sur leur droite ( $\phi$ croissants) et fonds dunaires sur leur gauche (cf. B.3.2),

- pour les écoulements obéissant aux lois du régime, la courbe limite notée $\left(L_{2}\right)$ sur la figure 1 sépare : à sa droite, un transport solide par charriage et suspension gouverné par les équations d'ENGELUND-HANSEN ; à sa gauche un transport solide par charriage seul gouverné par les équations de MEYer-PETER.

Nota: La courbe limite $\left(L_{1}\right)$, située à gauche de $\left(L_{2}\right)$, constituant l'asymptote de toutes les courbes iso $d$ (cf. A.3.3.3), tout point représentatif situé sur cette courbe correspondrait donc toujours à un écoulement « en régime » ainsi qu'à un transport solide par charriage.
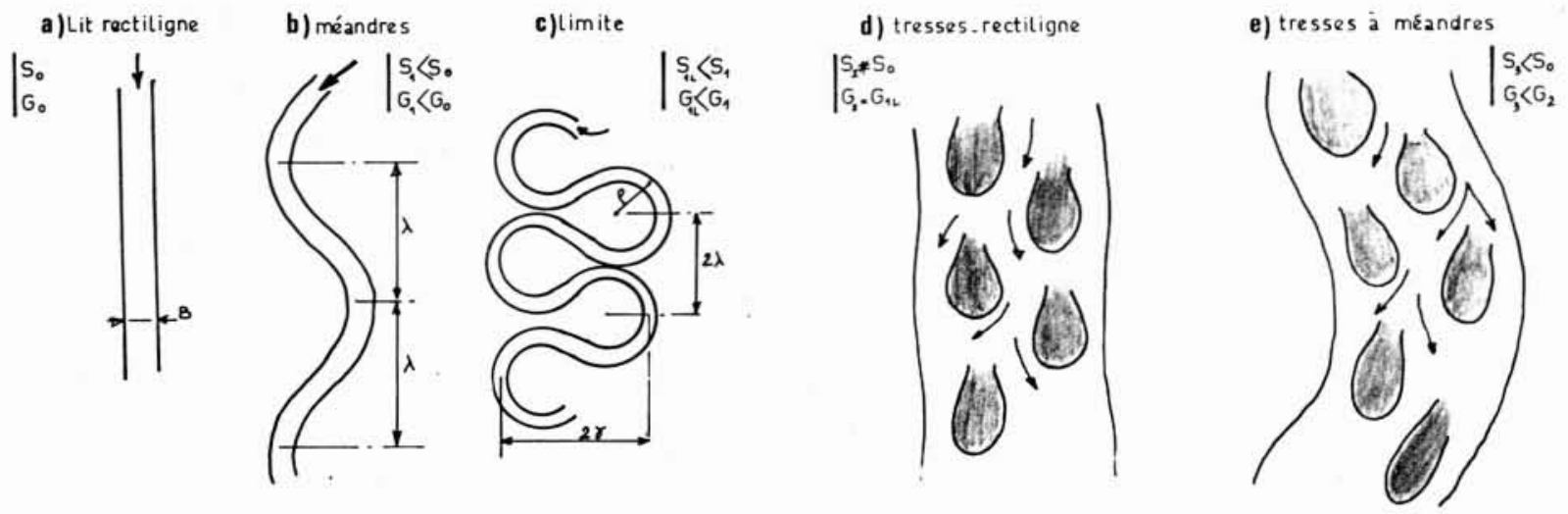

2.

3.

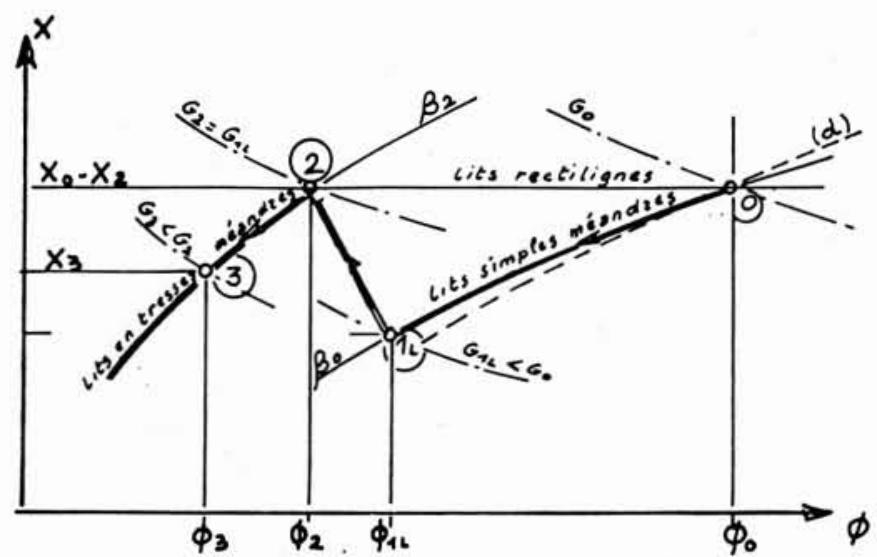




\section{B. MORPHOLOGIE DES COURS D'EAU HORS RÉGIME}

\section{B.1. Principes généraux}

L'ensemble des configurations morphologiques peut se résumer à 3 types principaux :

— lits rectilignes,

- lits à méandres,

- lits en tresses.

Dans l'optique des développements qui suivent, les lits à méandres et les lits en tresses correspondent à une " dégénérescence " des lits rectilignes (obéissant aux lois du régime). Le paramètre moteur de cette dégénérescence est la décroissance du débit solide par rapport à la valeur maximale initiale $\left(G_{0}\right)$ correspondant au régime.

Les schémas de la figure 2 suggèrent les différentes étapes possibles de l'évolution d'un lit à partir d'une configuration rectiligne; la limite de la configuration en méandres apparaît lorsque ces méandres se "courtcircuitent ", donnant alors naissance aux lits en tresses. Au cours de l'évolution des méandres, la pente diminue jusqu'à une valeur minimale correspondant à la limite de leur court-circuit, puis augmente brutalement pour retrouver une valeur proche de celle du lit rectiligne initial.

\section{B.2. Hypothèses de base}

\section{B.2.1. Concernant la configuration en méandres}

Le débit $Q$ et la dimension $d$ du sédiment déterminent les caractéristiques du lit rectiligne obéissant aux lois du régime, c'est-à-dire aux équations de LACEY. La prise en compte d'un écoulement sur fond plat permet de déterminer la pente $S_{0}$ correspondante.

Sur la figure 1 le point représentatif $X_{0}, \phi_{0}$ du lit en " régime " est déterminé par l'intersection des courbes iso $\beta$ et iso $d$ (avec $\left(\frac{\beta+2}{\beta}\right)^{2} \beta=38 Q^{1 / 6} d^{1 / 6}$ ).

A ce point représentatif correspond une valeur du débit solide $G_{0}$ déterminé par la valeur correspondante de :

$Y_{01}=G_{0} S_{0}^{5 / 4} / d^{5 / 2}$ si le transport solide est du type charriage et suspension (à droite de la limite $L_{2}$ ) ou par la valeur de :

$Y_{11}=G_{0} S_{0} / d^{5 / 2}$ si le transport solide s'opère par charriage seul (à gauche de la limite $L_{2}$ ).
La configuration en méandres apparaît si $S_{1}<S_{0}$; le lit doit en effet allonger son cours pour diminuer sa pente (figure $2 b$ ).

Dans cette configuration en méandres on fait l'hypothèse que la valeur de $\beta$ garde la valeur initiale : $\beta_{1}=\beta_{0}$.

Pour $Q$ et $d$ donnés, le point représentatif $X_{1}$, $\phi_{1}$ du méandre se déplace donc sur la courbe iso $\beta=\beta_{0}$ et $X_{1}<X_{0}$ (puisque $S_{1}<S_{0}$ ) de même $\phi_{1}<\phi_{0}$; les valeurs de $Y_{01}$ (ou $Y_{11}$ ) correspondantes diminuent elles aussi et on vérifiera aisément qu'il en est de même pour le débit solide: $G_{1}<G_{0}$.

Inversement toute diminution du débit solide par rapport à sa valeur maximum maximorum, $G_{0}$, se traduira par une diminution de la pente et donc par l'apparition de méandres.

La figure 1 montre que la courbe iso $\beta$ sur laquelle se déplace le point représentatif $\left(X_{1}, \phi_{1}\right)$ se situe à gauche de la courbe iso $d$ mais en est relativement proche ; on supposera en première approximation que ce point se situe dans la frange d'incertitude où les fonds peuvent encore être considérés comme plats (cf. A.3.2.1).

\section{B.2.2. Concernant la configuration lits en tresses}

Dès que la valeur de $S_{1}$ atteint une valeur suffisamment faible il y a court-circuit des méandres, la pente retrouve une valeur proche de la valeur initiale (figure $2 d$ ) :

$$
S_{2} \# S_{0} \text {. }
$$

Le débit solide est évidemment pratiquement égal à celui immédiatement avant court-circuit :

$$
G_{2}=G_{1 L}
$$

et l'allongement $\beta$ croît brutalement :

$$
\beta_{2} \gg \beta_{1}=\beta_{0} \text {. }
$$

Le point représentatif $\left(X_{2} \# X_{0}, \phi_{2}\right)$ de l'écoulement après court-circuit se situe largement à gauche de la courbe iso $d$ (ou iso $\beta$ ) correspondante et traduit de ce fait généralement un transport solide sur dunes et non plus sur fonds plats.

Si le débit solide continue de décroître: $G_{3}<G_{2}$ le point représentatif $X_{3}, \phi_{3}$ se déplace sur la courbe iso $\beta_{2}=\beta_{3}$ et on retrouve un processus de méandrement $\mathrm{du}$ lit en tresses (figure 2e).

Les considérations exposées en B.2.1 et B.2.2 sont traduites sur le schéma de la figure 3 . 
4.
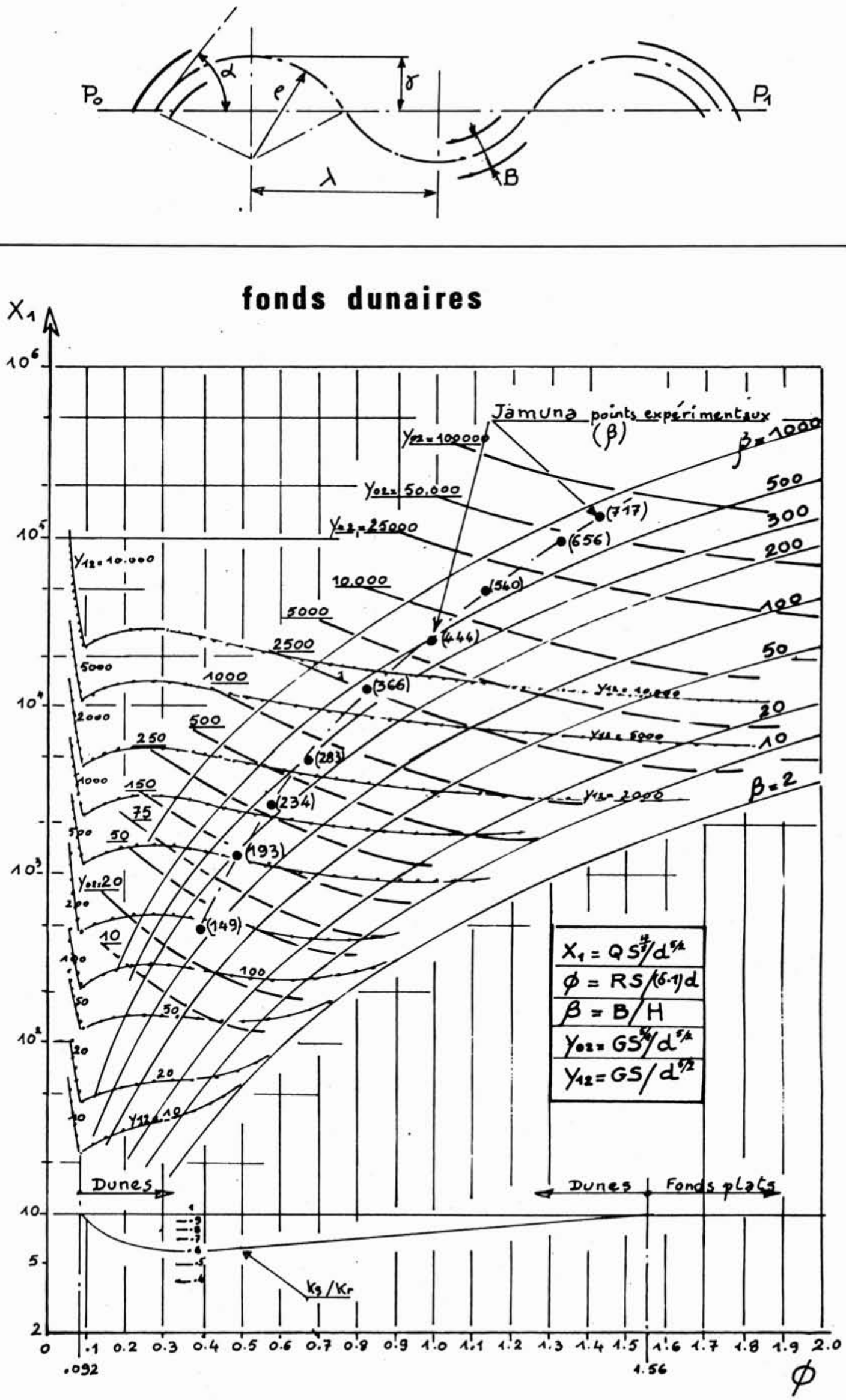


\section{B.3. Quantification des hypothèses émises}

\section{B.3.1. Schématisation des méandres (figure 4)}

Les méandres sont assimilés à des séries d'arcs de cercles alternés autour de la ligne $P_{0}, P_{1}$ de plus grande pente.

Soit $\alpha$ l'angle (en radians) que fait la tangente au méandre avec la ligne de plus grande pente $P_{0}$, $P_{1}$.

- $S_{0}$ la plus grande pente,

- $B$ la largeur du lit,

- $\rho$ le rayon de courbure du méandre que des corrélations expérimentales évaluent entre 2,3 et $3 \mathrm{~B}$.

Des considérations géométriques simples, à partir du schéma de la figure 4, permettent d'évaluer la pente $S_{1}$ du lit, la demi-amplitude du méandre $\gamma$, sa demilongueur d'onde $\lambda$, en fonction de l'angle $\alpha$.

Avec $\rho=3 B$ on trouve :

$$
\mid \begin{aligned}
& S_{1}=S_{0} \sin \alpha / \alpha \\
& \gamma=3 B(1-\cos \alpha) \\
& \lambda=6 B \sin \alpha .
\end{aligned}
$$

\section{Exemples}

- Pour $\alpha=\pi / 2$ rad :

$$
\begin{aligned}
& S_{1}=0,64 S_{0} \\
& \gamma=3 B \\
& \lambda=6 B
\end{aligned}
$$

- La configuration limite des méandres est telle que (cf. figure 2c) :

$$
2 \rho+B=2 \lambda
$$

On en déduit, à partir des relations (XIV) ;

$$
\begin{gathered}
\sin \alpha=7 / 12, \alpha=2,52 \operatorname{rad}\left(144^{\circ}\right) \\
S_{1}=0,23 S_{0} \\
\gamma=5,4 B \\
\lambda=3,5 B .
\end{gathered}
$$

Nota: Les deux exemples donnés constituent probablement les configurations extrêmes du domaine à l'intérieur duquel les risques de court-circuit des méandres existent ; pratiquement $S_{1}=0,4$ à $0,5 S_{0}$ pourrait constituer un domaine moyen où ce risque doit être pris en considération $\left(108^{\circ}<\alpha<122^{\circ}\right)$.

\section{B.3.2. Coefficient de rugosité en présence de dunes}

Les expériences d'ENGELUND-HANSEN dans le domaine des dunes (réf. $d$ ), traduites analytiquement par $\mathrm{CHOL}$ LET et CUNGE (réf. $e$ ), peuvent s'exprimer par la relation suivante donnant le rapport entre le coefficient de rugosité avec dunes $\left(k_{s}\right)$ et le coefficient de rugosité sur fond plat $\left(k_{r}\right)$ en fonction de $\phi$

$$
k_{s} / k_{r} \# 1,3\left[\frac{0,06+0,41 \phi^{2}}{\phi}\right]^{5 / 8} .
$$

Cette relation est représentée sur la figure 5 .
Les dunes apparaissent en principe dans tout le domaine où $k_{s} / k_{r}<1$, c'est-à-dire pour :

$$
0,092<\phi<1,56
$$

En fait le domaine d'apparition des dunes est plus restreint si l'on se réfère aux conclusions tirées de l'examen des équations au régime, puisque les dunes n'apparaissent que pour des points représentatifs situés à gauche des courbes iso $d$.

On remarquera que la relation $k_{s} / k_{r}$ présente des valeurs minimales proches de 0,64 pour $0,25<\phi<0,55$; dans ce domaine la rugosité est donc maximale de même que la hauteur relative des dunes.

\section{B.3.3. Equation de l'écoulement en présence de dunes}

L'équation de MANNING-STRICKLER sur fonds de dunes s'exprime de la façon suivante :

$$
\begin{aligned}
X_{1} & =Q S^{17 / 8} / d^{5 / 2} \\
& =91,4 \phi^{2}\left(0,06+0,41 \phi^{2}\right)^{5 / 8}\left(\frac{\beta+2}{\beta}\right)^{47 / 24} \cdot \beta
\end{aligned}
$$

Cette relation est représentée sur la figure 5 dans les axes $X_{1}, \phi$, avec $\beta$ comme paramètre.

\section{B.3.4. Equations du transport solide sur dunes}

\section{B.3.4.1. Domaine du charriage et suspension}

L'équation d'ENGELUND-HANSEN exprimée avec les paramètres $Y, \phi, \beta$ s'écrit :

$$
\begin{aligned}
Y_{02} & =G S^{5 / 4} / d^{5 / 2} \\
& =40,3 \phi^{5 / 2}\left(0,06+0,41 \phi^{2}\right)^{5 / 4}\left(\frac{\beta+2}{\beta}\right)^{11 / 12} \cdot \beta
\end{aligned}
$$

\section{B.3.4.2. Domaine du charriage}

L'équation de MEYER-PETER-MÜLleR s'écrit :

$$
\begin{aligned}
Y_{12}=G S / d^{5 / 2}=72 \phi & {\left[1,426\left(\frac{0,06+0,41 \phi^{2}}{\phi}\right)^{15 / 16}\right.} \\
& . \phi-0,047]^{3 / 2}(\beta+2)
\end{aligned}
$$

Les courbes $Y_{02}=$ cste et $Y_{12}=$ cste sont représentées dans les axes $X_{1}, \phi$ sur la figure 5 .

On notera que, hors du domaine d'apparition des dunes, les relations : $X_{1}(\phi, \beta), Y_{02}\left(X_{1}, \phi\right), Y_{12}\left(X_{1}, \phi\right)$, coïncident évidemment avec celles établies sur fond plat (figure 1). 
Tableau B

\begin{tabular}{|c|c|c|c|c|c|c|c|c|c|c|c|}
\hline$\phi_{\mathrm{L}}$ & 0,1 & 0,15 & 0,2 & 0,25 & 0,3 & 0,35 & 0,4 & 0,5 & 0,6 & 0,7 & 0,8 \\
\hline$S_{L}$ & $10^{-5}$ & $610^{-5}$ & $1,610^{-4}$ & $3,310^{-4}$ & $5,810^{-4}$ & $9,110^{-4}$ & $1,310^{-3}$ & $2,610^{-3}$ & $4,710^{-3}$ & $7,910^{-3}$ & $1,210^{-2}$ \\
\hline
\end{tabular}

\section{B.3.4.3. Domaines d'application des équations d'ENGELUND-HANSEN et de MEYER-PETER}

La courbe $\left(L_{2}\right)$ de la figure 1 limite les domaines d'application des formules d'ENGELUND-HANSEN et MEYER-PETER pour les écoulements sur fonds plats obéissant aux lois du régime.

De même pour les écoulements hors régime sur dunes, les équations (4.0.2) et (4.1.2) permettent de déterminer la relation entre $S$ et $\phi$ pour que la valeur $G$ du débit solide soit identique avec l'une ou l'autre des formules. En remarquant que

$$
\left(\frac{\beta+2}{\beta}\right)^{11 / 12} \cdot \beta \# \beta+2
$$

on établit aisément les relations données dans le tableau $B ; \phi$ et $S$ sont indicés $(L)$ pour indiquer qu'il s'agit de valeurs limites.

A un écoulement de pente $S=S_{L}$ correspondra un transport solide par charriage plus important si le point représentatif dans les axes $X, \phi$ est tel que $\phi<\phi_{L}$.

Inversement si $\phi>\phi_{L}$, le transport solide par charriage et suspension donné par la formule d'ENGELUNDHANSEN sera plus important.

Par exemple, si $S=1,610^{-4}$ et $\phi>\phi_{L}=0,2$ le transport solide par charriage et suspension est prépondérant.

\section{B.4. Application des principes et hypothèses}

\section{B.4.1. Domaine charriage et suspension}

\section{B.4.1.1. Régime et méandres}

L'écoulement dans des lits simples à méandres a été supposé s'effectuer sur fond plat comme pour les écoulements obéissant aux lois du régime.

Régime: La combinaison des équations générales (3.0) : écoulement sur fond plat, et (4.0.1) : transport solide par charriage et suspension, sur fonds plats, avec $\beta$ obéissant aux équations du régime :

$$
\left(\frac{\beta+2}{\beta}\right)^{2} \cdot \beta=38 Q^{1 / 6} d^{1 / 6}
$$

permet d'établir la relation approchée suivante liant : le débit $Q$, la dimension $d$, du sédiment, la pente $S_{0}$ et le transport solide $G_{0}$ correspondant au régime.

$$
G_{0} \# 1,11.10^{-2} Q^{1,36} S_{0}^{1,785} / d^{1.138}
$$

Cette relation est évidemment équivalente à la relation (XI) donnée précédemment.
Méandres: L'allongement $\beta_{1}$ du lit à méandres étant supposé identique à celui du régime $\beta_{1}=\beta_{0}$, pour des mêmes valeurs de $Q$ et $d$, le transport solide $G_{1}$ sera tel que :

$$
G_{1}=G_{0}\left(S_{1} / S_{0}\right)^{1.785} \text {. }
$$

A la limite du court-circuit des méandres, c'est-à-dire pour $0,4<S_{1} / S_{0}<0,5$ (cf. B.3.1 Nota) l'expression (XVII) conduit à :

$$
0,2<G_{1 L} / G_{0}<0,3 \text {. }
$$

\section{B.4.1.2. Lits en tresses}

Après court-circuit des méandres (cf. figures $2 c$ et $2 d$ ) l'allongement $\beta$ croît brutalement : $\beta_{2} \gg \beta_{1}=\beta_{0}$, l'écoulement se fait sur fonds dunaires, la pente retrouve sensiblement sa valeur initiale : $S_{2} \# S_{0}$, le débit solide est égal à celui du lit à méandres avant court-circuit : $G_{2}=G_{1 L}$; pour ne pas alourdir ce qui suit on suppose que $G_{1 L}=0,3 G_{0}$ :

$$
G_{2}=0,3 G_{0}
$$

La combinaison de l'équation générale de l'écoulement sur fonds dunaires : équation (3.1) et de l'équation du transport solide (4.0.2), dans laquelle on introduit la valeur de $G=G_{2}=0,3 G_{0}$ permet le calcul approché de $\beta_{2}$ :

$$
\beta_{2} \# 89 Q^{0.29} S_{0}^{0.333} / d^{0.254} \text {. }
$$

Il est alors possible d'exprimer la largeur $B$ du lit et le rayon hydraulique $R \# H$ en fonction de $Q, S$ et $d$; on trouve les relations suivantes :

$$
\begin{aligned}
& R \# 9,510^{-2} Q^{0.233} d^{0.26} / S^{0.41} \\
& B \# 8,4 Q^{0.527} d^{0.006} / S^{0.077} .
\end{aligned}
$$

\section{Comparaison avec l'expérience}

A ce stade, il est évidemment important de comparer les relations théoriques établies pour les lits en tresses avec des relations expérimentales. Pour ce faire, nous nous 
référons aux études morphologiques entreprises sur la Jamuna, au Bangladesh, par une équipe du Laboratoire d'Hydraulique de Delft (réf. $f$ ). Dans la zone étudiée, $d$ est de 0,18 à $0,210^{-3} \mathrm{~m}$ et $S$ de 6 à $710^{-5}$.

a) Caractéristiques géométriques des lits: Les points représentatifs des corrélations expérimentales établies par Delft pour des débits compris entre $4000 \mathrm{~m}^{3} / \mathrm{s}$ et $44000 \mathrm{~m}^{3} / \mathrm{s}$ sont portés dans les axes $X, \phi$ de la figure 5 avec, en regard la valeur de $\beta$ correspondante; on constate que l'accord général entre expérience et théorie des écoulements sur fonds dunaires est satisfaisant. Par ailleurs, tous les points expérimenaux étant tels que pour $S=610^{-5}$, les valeurs de $\phi$ sont toujours plus grandes que $\phi_{L}$ (cf. tableau $B$ ), le transport solide s'effectue donc par charriage et suspension.

Mises sous forme analytique, les corrélations expérimentales données par Delft sont les suivantes :

$$
\mid \begin{aligned}
& R=0,56 Q^{0.23} \\
& B=18,9 Q^{0.51} \\
& \beta=33,75 Q^{0.28} .
\end{aligned} .
$$

Avec les valeurs moyennes de $S=610^{-5}$ et $d=0,1810^{-3} \mathrm{~m}$ les relations théoriques $(\mathbf{X X}),(\mathbf{X X I})$ et (XXII) s'écrivent :

$$
\mid \begin{aligned}
& R=0,54 Q^{0,233} \\
& B=16,9 Q^{0,527} \\
& \beta=31,3 Q^{0.294}
\end{aligned} .
$$

L'accord entre théorie et expérience est donc assez satisfaisant.

b) Relation entre la pente et le sédiment: Il était intéressant d'appliquer les relations (VIII) ou (IX), liant théoriquement $S, d$ et $Q$ pour les écoulements obéissant aux lois du régime, aux écoulements sur lit en tresses rectilignes ; ces relations devraient en effet être équivalentes puisque $S_{2} \# S_{0}$ et que $d$ reste évidemment une constante.

Avec $S=610^{-5}, d=0,1810^{-3}$ et $Q$ compris entre $44000 \mathrm{~m}^{3 /} / \mathrm{s}$ et $4000 \mathrm{~m}^{3} / \mathrm{s}$, l'expression :

$$
d^{0.151} /\left(S^{0.19} Q^{0.016}\right)=E
$$

satisfait à l'inégalité suivante

$$
1,45<E<1,51 .
$$

Les relations (VIII) et (IX) donnent une valeur de $E$ comprise entre 1,48 pour les faibles valeurs de $\beta$ et 1,61 pour les fortes valeurs de $\beta$.

Remarquons que le calcul de $\beta_{0}$ correspondant au régime pour la Jamuna, avec

$$
\left(\frac{\beta_{0}+2}{\beta}\right)^{2} \cdot \beta=38 Q^{1 / 6} d^{1 / 6}
$$

donne, pour les valeurs extrêmes de $Q$ envisagées :

$$
32<\beta_{0}<50 .
$$

Ce sont des valeurs de $\beta$ relativement faibles qui conduiraient plutôt à adopter $E=1,48$ et dans ce cas l'accord théorie-expérience serait assez correct. c) Transport solide: Le transport solide dans des lits en tresses a été évalué à $0,3 G_{0}$, soit d'après (XVI) :

$$
G_{2}=0,3310^{-2} Q^{1,36} S_{0}^{1.785} / d^{1.138} \text {. }
$$

(XXIII)

Avec les données prises en compte pour la Jamuna, soit $S=610^{-5}$ et $d=0,1810^{-3} \mathrm{~m}$, l'expression (XXIII) s'écrit :

$$
G_{2}=1,7510^{-6} Q^{1,36} \mathrm{~m}^{3} / \mathrm{s}
$$

avec $S=710^{-5}$ on trouverait :

$$
G_{2}=2,310^{-6} Q^{1.36} \mathrm{~m}^{3} / \mathrm{s} \text {. }
$$

Les données acquises au cours de campagnes de mesures réalisées pendant la période 1968-1970 ont conduit à la relation suivante en ce qui concerne le transport en suspension (réf. $g$ ) :

$$
G_{2}=4,110^{-6} Q^{1.38} \mathrm{~m}^{3} / \mathrm{s} .
$$

(XXVI)

L'article (réf. $g$ ) dans sa conclusion, indique que "The ENGELUND-HANSEN transport formula multiplied by 2 gives a faire prediction of the bed material transport in the Jamuna River ".

C'est bien sensiblement à la même conclusion que l'on arrive si l'on compare les relations théoriques (XXIV) ou (XXV) avec la relation expérimentale (XXVI).

Des campagnes de mesures ont été entreprises plus récemment entre mai 1982 et juin 1984 (réf. $h$ ). Ces campagnes sont traduites sur la figure 6 (page suiv.); elle donne les transports solides en $t / j$ pour diverses valeurs de $d$ en fonction du débit en $\mathrm{m}^{3} / \mathrm{s}$. La relation (XXIV), transcrite en $\mathrm{t} / \mathrm{j}$ avec $1 \mathrm{~m}^{3} \# 2 \mathrm{t}$, s'écrit :

$$
G_{2} \# 0,3 Q^{1,36} \mathrm{t} / \mathrm{j}
$$

(XXIVbis)

elle est représentée sur cette même figure 6 .

On constate que l'accord entre cette relation théorique et les mesures est satisfaisant en ce qui concerne le transport solide de la fraction des sables ayant un diamètre moyen égal ou plus grand que $0,06210^{-3} \mathrm{~m}$ ( $1 / 3 \mathrm{de} \mathrm{dm})$; elle diverge par contre, dans un rapport de 2 en moyenne, si l'on prend en compte la totalité du transport en suspension incluant les fractions les plus fines du sable.

La formule d'ENGELUND-HANSEN donnerait donc des résultats corrects si le pourcentage des sables, dont le diamètre est inférieur à environ $1 / 3 \mathrm{de} \mathrm{dm}$, était négligeable. S'il n'en est pas ainsi, l'erreur peut conduire à une sous-estimation du transport solide dans un rapport qui peut atteindre 2 ou davantage ; c'est le cas de la Jamuna.

On remarquera que le point expérimental porté sur la figure 5 et correspondant à un débit de $55000 \mathrm{~m}^{3} / \mathrm{s}$ a une abscisse $\phi=1,45$; c'est une valeur très proche de celle 


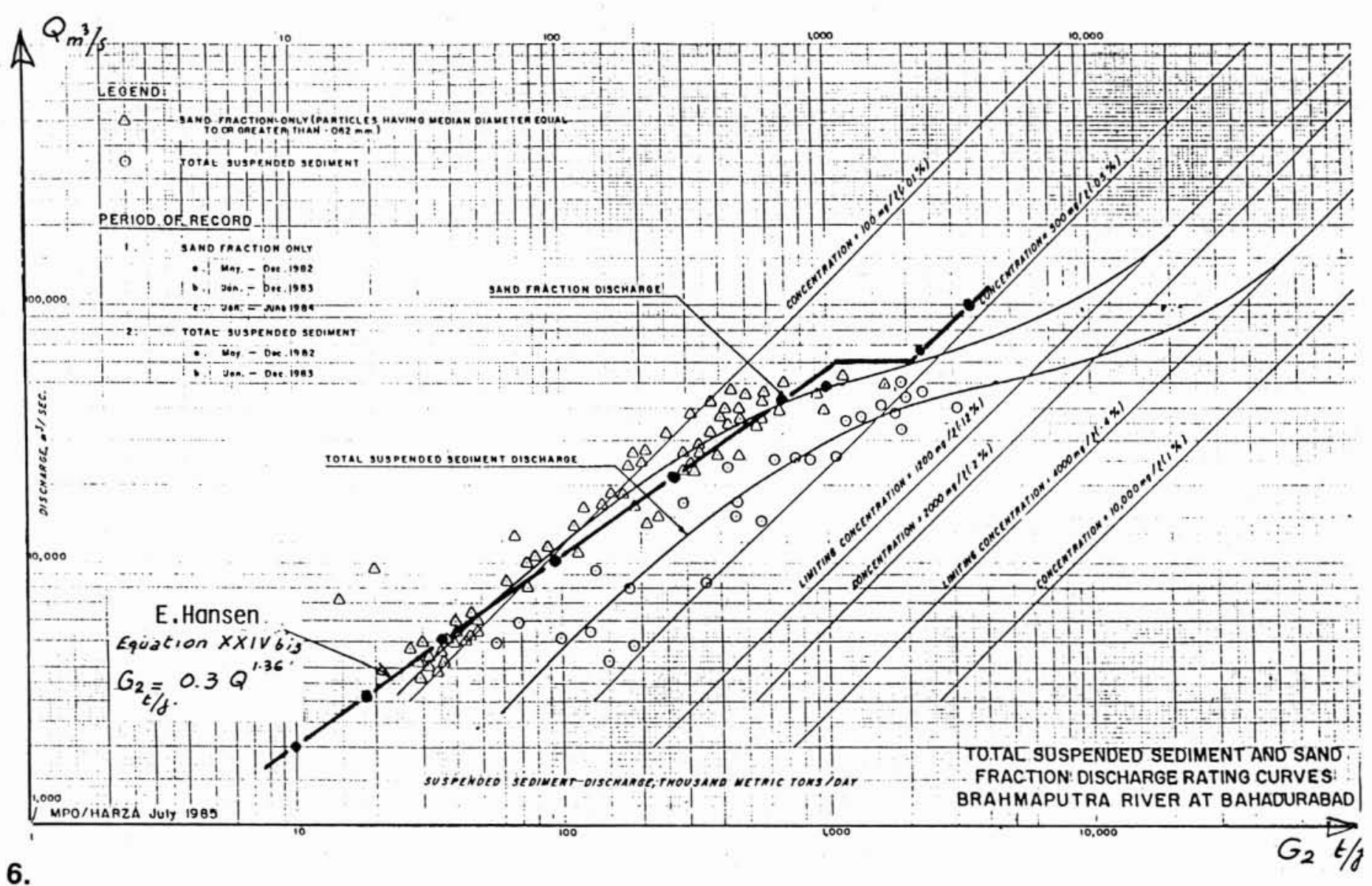

au-delà de laquelle les dunes disparaissent $(\phi=1,56-$ cf. B.3.2).

Les fonds plats sont effectivement observés dans la Jamuna lorsque le débit atteint des valeurs de $60000 \mathrm{~m}^{3} / \mathrm{s}$ et au-delà.

La relation $G_{2}(Q)$ de la figure 6 a été prolongée audelà de $60000 \mathrm{~m}^{3} / \mathrm{s}$ en tenant compte de cette remarque.

Les interprétations théoriques concernant les données expérimentales de la Jamuna ont été développées dans le rapport (réf. $i$ ).

d) Remarques:

- Les relations établies précédemment pour les lits en tresses sont valables uniquement après court-circuit du méandre, c'est-à-dire pour $G_{2} \# 0,3 G_{0}$; on est alors en présence d'un lit en tresses, de grand allongement $\beta_{2}$, et pratiquement rectiligne ; c'est le cas de la Jamuna (figure $2 d$ ).

Si le débit solide continue de décroître : $G_{3}<G_{2}$ on retrouve alors un processus de méandrement (figure $2 e$ ). Ce type de lit en tresses, très large et qui présente des méandres est assez souvent observé; le lit du Gange dans sa traversée du Bangladesh en est un exemple.

Le point représentatif $X_{3}, \phi_{3}$ de ce type de lit est porté sur le schéma de la figure 3.
- Le paragraphe (b) précédent montre que le rapport $E=d^{0,151} /\left(S^{0,19} Q^{0,016}\right)$ est en moyenne de 1,48 pour la Jamuna, c'est-à-dire identique à la valeur théorique pour les valeurs faibles de $\beta$. On est donc tenté de généraliser cette remarque et, dans ce cas, les relations établies précédemment exprimant $\beta, R, B$ et $G$ pourraient être écrites en éliminant $S$ ou $d$.

\section{B.4.2. Domaine du charriage seul}

\section{B.4.2.1. Régime et méandres}

Par définition, dans ces deux cas, le charriage s'opère sur fonds plats.

Régime: Rappelons que les points représentatifs des écoulements obéissant aux lois du régime et dont le transport solide s'effectue par charriage seul se situent à gauche de la limite $\left(L_{2}\right)$ représentée sur la figure 1 .

La combinaison de l'équation (3.0) : écoulement sur fonds plats, et (4.1.1): transport solide par charriage seul sur fond plat, avec $\beta$, obéissant aux équations du régime :

$$
\left(\frac{\beta+2}{\beta}\right)^{2} \cdot \beta=38 Q^{1 / 6} d^{1 / 6}
$$


permettrait d'établir théoriquement la relation liant le transport solide $G_{0}$, au débit, à la pente et au sédiment.

En fait, il est beaucoup plus simple, à partir de la figure 1 , d'observer qu'entre les limites $\left(L_{2}\right)$ et $\left(L_{1}\right)$, pour une même valeur de $X_{0}=Q S^{17 / 8} / d^{5 / 2}$, la valeur de $Y_{11}=G_{0} S / d^{5 / 2}$ reste pratiquement constante et est liée à $X$ par la relation approchée suivante :

$$
X_{0} \# 1,33 Y_{11}^{0.980}
$$

(XXVII)

on en déduit :

$$
G_{0} \# 0,75 Q^{1,02} S_{0}^{1,17} / d^{0,05}
$$

(XXVIII)

Cette relation est équivalente à la relation (XII).

Méandres : L'allongement $\beta_{1}$ du lit à méandres étant par hypothèses identique à celui du régime $\left(\beta_{1}=\beta_{0}\right)$, pour des mêmes valeurs de $Q$ et $d$, le transport solide se déduit de la relation (XXVIII) :

$$
G_{1}=G_{0}\left(S_{1} / S_{0}\right)^{1.17}
$$

$(\mathbf{X X I X )}$

A la limite du circuit des méandres, en adoptant $S_{1} / S_{0}=0,5$ on trouve :

$$
G_{1 L}=0,44 G_{0}
$$

\section{B.4.2.2. Lits en tresses}

Le lit en tresses apparaît après court-circuit des méandres, $\beta$ passe de $\beta_{1}=\beta_{0}$ à $\beta_{2}$, la pente retrouve sa valeur initiale : $S_{2} \# S_{0}$, le débit solide sur fonds dunaires est égal à $G_{2}=G_{1 L}$ soit, d'après $(\mathbf{X X V I I I )}$ et $(\mathbf{X X X})$

$$
G_{2}=0,33 Q^{1.02} S_{0}^{1.17} / d^{0.05}
$$

$(\mathbf{X X X I})$

Evaluation des valeurs de $\beta, B, R$ : Il est toujours possible de faire une évaluation à partir des équations de l'écoulement sur dunes : équation (3.1) et du charriage en présence de dunes: équation (4.1.2).

Mais ces équations ne sont pas aisées à mettre sous une forme analytique simple et il est sans doute plus commode de raisonner graphiquement à partir d'exemples.

\section{Exemple 1}

Soit $Q=2000 \mathrm{~m}^{3} / \mathrm{s}, d=510^{-3} \mathrm{~m}, S=6,410^{-4}$.

Les caractéristiques du lit obéissant aux équations du régime sont telles que

$$
\left(\frac{\beta+2}{\beta}\right)^{2} \cdot \beta=38(2000)^{1 / 6}\left(510^{-3}\right)^{1 / 6}
$$

on en déduit: $\beta_{0} \# 52$.
La valeur de $X_{0}$ correspondante $=Q S^{17 / 8} / d^{5 / 2}=185$.

Le point représentatif $\left(A_{0}\right)$ du régime est porté sur le schéma de la figure 7 , qui reproduit partiellement la figure 1 (fonds plats) ; ce point représentatif est à gauche de la limite $\left(L_{2}\right)$ et on vérifie donc que le transport solide s'effectue bien par charriage.

La relation (XXVII) liant $X_{0}$ et $Y_{11}=G_{0} S_{0} / d^{5 / 2}$ permet d'évaluer $Y_{11}$

$$
Y_{11}=154
$$

on en déduit $G_{0}=0,42 \mathrm{~m}^{3} / \mathrm{s}$.

A la limite de coupure du méandre qui est généré si $G<G_{0}: G_{1 L}=0,44 G_{0}, S_{1}=0,5 S_{0}$, le point représentatif $(A 1)$ représenté sur la figure 7 est tel que:

$$
\begin{aligned}
& \beta_{1}=\beta_{0}=52 \\
& X_{1}=42,4 \\
& Y_{11}=34 .
\end{aligned}
$$

Le débit solide correspondant est $G_{1 L}=0,18 \mathrm{~m}^{3} / \mathrm{s}$.

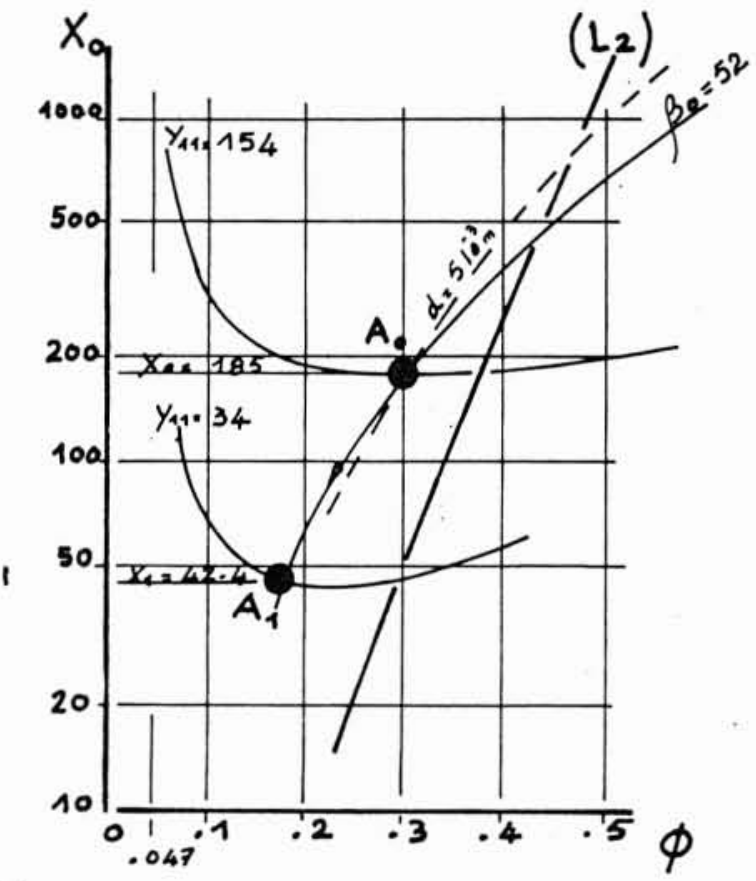

7.

Après coupure du méandre, avec $S_{2} \# S_{0}, X_{2} \# X_{0}$, $G_{2}=G_{1 L}=0,44 G_{0}$, la valeur de $Y_{12}$ correspondant au transport solide sur dunes est égale à $0,44 Y_{11}$

$$
Y_{12}=68
$$

et $G_{2}=G_{1 L}=0,18 \mathrm{~m}^{3} / \mathrm{s}$. 
Le point représentatif $\left(A_{2}\right)$ :

$$
\mid \begin{aligned}
& Y_{2}=185 \\
& Y_{12}=68
\end{aligned}
$$

est porté sur le schéma de la figure 8 qui reproduit partiellement la figure 5 (fonds dunaires). Il y correspond une valeur de $\beta_{2}$ qui peut être déterminée graphiquement ou par le calcul; on trouvera $\beta_{2} \# 1000$.

On est là en présence d'un lit très large dans lequel les dunes sont pratiquement inexistantes puisque $\phi_{2} \# 0,09$ correspond à la limite d'apparition des dunes.

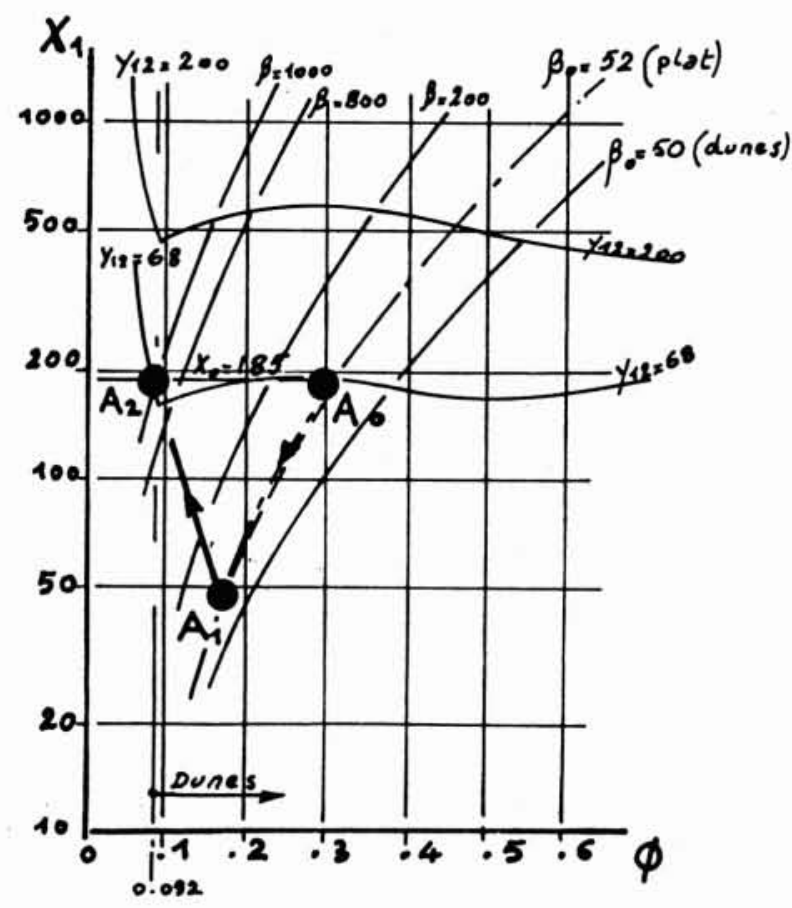

8.

\section{Exemple 2}

Cet exemple correspond à un écoulement obéissant aux équations du régime mais dont le point représentatif se situe sur la courbe limite $\left(L_{1}\right)$ représentée sur la figure 1 .

Soit $\quad Q=3000 \mathrm{~m}^{3} / \mathrm{s}, d=0,21 \mathrm{~m}, S=2 \%$.

A ces valeurs correspondent :

$$
\begin{aligned}
& X_{0}=36 \\
& \phi_{0} \# 0,17 \\
& \beta_{0} \# 50
\end{aligned}
$$

et d'après l'équation (XXVII)

$$
Y_{11} \# 29
$$

on en déduit : $G_{0} \# 29 \mathrm{~m}^{3} / \mathrm{s}$.
Ce type d'écoulement en "régime ", c'est-à-dire sur fonds plats, apparaît toujours lorsque le point représentatif $X_{0}$ sur la courbe limite $\left(L_{1}\right)$ se situe en dessous de la valeur de $X$, correspondant à la jonction asymptotique de la courbe iso $(d)$ avec $\left(L_{1}\right)$, ce qui est bien le cas dans l'exemple donné.

Cet exemple correspond au cas réel de la « Rivière des Galets ", située dans l'île de la Réunion. Cette rivière a fait l'objet d'études sur modèle réduit à l'échelle 1/120 qui ont permis, en particulier, de déterminer la loi de variation :

$$
G_{0} \mathrm{~m}^{3} / \mathrm{s}=f(Q) .
$$

La densité du sédiment de la Rivière des Galets est cependant plus forte que celle prise en compte dans les développements théoriques qui précèdent puisqu'elle est de $\partial=2,8$ au lieu de $\partial=2,6$.

$\mathrm{La}$ formule initiale de MEYER-PETER exprime le transport solide non pas en volume mais en poids pesé sous l'eau. La transposition en volume dans les formules théoriques proposées avec $\partial=2,6$ a donc été faite en divisant le poids pesé sous l'eau par $(2,6-1)=1,6$ et en multipliant par un coefficient de «foisonnement " $(f)$; avec $\partial=2,8$ le poids pesé sous l'eau devrait être divisé par $(2,8-1)=1,8$ multiplié par le même coefficient $(f)$.

Une formule du type (XXVIII) qui exprime $G_{0}$ avec $\partial=2,6$ doit donc être minorée dans le rapport $1,6 / 1,8$ pour exprimer $G_{0}$ avec $\partial=2,8$, soit :

$$
G_{0}=0,66 Q^{1.02} S_{0}^{1.17} / d^{0.05}
$$

(XXVIIIbis)

avec $S=2 \%$ et $d=0,21 \mathrm{~m}$ on trouve :

$$
G_{0}=7,310^{-3} Q^{1,02}
$$

pour $Q=3000 \mathrm{~m}^{3} / \mathrm{s}$ l'expérience donne : $G_{0} \# 19 \mathrm{~m}^{3} / \mathrm{s}$, la relation théorique précédente : $G_{0}=25,7 \mathrm{~m}^{3} / \mathrm{s}$.

La prise en compte, d'une part d'une fraction de l'ordre de 85 à $90 \%$ du débit qui participerait effectivement au charriage et d'autre part d'un débit de seuil de charriage, non nul, permet d'améliorer sensiblement la comparaison théorie-expérience.

L'étude de la Rivière des Galets est développée dans le rapport (réf. $\jmath$ ).

Nota: Pour $Q$ variant de 100 à $3000 \mathrm{~m}^{3} / \mathrm{s}$ le rapport $E=d^{0,151} /\left(S^{0,19} Q^{0,016}\right)$ satisfait à l'inégalité suivante :

$$
1,46<E<1,54 \text {. }
$$

Cette inégalité est à comparer avec celle établie pour la Jamuna et donnée au chapitre B.4.1.2 § b :

$$
1,45<E<1,51 \text {. }
$$




\section{CONCLUSIONS}

La démarche qui consiste à supposer que les différentes morphologies des cours d'eau naturels découlent de la dégénérescence des cours d'eau rectilignes obéissant aux lois du régime paraît assez fructueuse ; elle permet en effet de rendre compte assez correctement de la morphologie de cours d'eau aussi différents que sont la Jamuna $\left(Q \sim 50000 \mathrm{~m}^{3} / \mathrm{s}, \quad d=0,1810^{-3} \mathrm{~m}, S=610^{-5}\right)$ et la Rivière des Galets $\left(Q \sim 3000 \mathrm{~m}^{3} / \mathrm{s}, d=0,21 \mathrm{~m}\right.$, $S=210^{-2}$ ).

Le paramètre « moteur " qui fait passer le lit rectiligne au lit à méandres puis au lit en tresses est la décroissance du débit solide ; en d'autres termes, le lit en tresses serait la conséquence d'un déficit en apport solide et non pas d'un excédent d'apport solide comme cela est très souvent affirmé.

Les chapitres qui précèdent devraient permettre, d'une part de mieux comprendre le «pourquoi » des morphologies de cours d'eau naturels et, d'autre part de comprendre le "comment " de la modification des morphologies induites par toute intervention artificielle : endiguement, augmentation du débit, diminution du débit solide par dragage... et qui se traduisent par des modifications de la pente, de la largeur moyenne, etc... c'est-à-dire par une nouvelle morphologie dont les caractéristiques asymptotiques pourront être évaluées.

Bien entendu, dans des développements futurs, le paramètre "temps" devra être pris en compte pour déterminer la durée de l'évolution morphologique permettant de passer de la situation initiale à la situation finale ; ce problème ne peut être traité qu'à l'aide d'un modèle mathématique reproduisant en particulier, les hydrogrammes $Q(t)$. Un tel modèle pourrait n'introduire que les données moyennes du lit : $\bar{B}, \bar{H}, \ldots$ L'exemple de la Jamuna, malgré la complexité du lit, semble en effet indiquer que la simplification que constitue la prise en compte de valeurs moyennes donne malgré tout des résultats acceptables.

Soulignons que, bien entendu, certaines hypothèses ou relations mériteront d'être vérifiées ou approfondies.

\section{$A$ propos du paramètre $E$}

Par exemple, le paramètre $E=d^{0,151} /\left(S^{0,19} \cdot Q^{0,016}\right)$ reste assez «ambigu ». Dire qu'il est constant, comme cela paraît se déduire de l'analyse des équations du régime et des équations théoriques de l'écoulement, supposerait que la pente $S$ est une fonction légèrement décroissante du débit $\left(S \sim Q^{-0,08}\right)$.

En fait, cette conclusion n'est pas en accord avec l'observation car il semble bien que dans un cours d'eau (exclusion faite des phénomènes dus à la non-permanence de l'écoulement) la pente reste sensiblement constante, c'est d'ailleurs ce qui a été supposé dans les exemples donnés précédemment.

Cette ambiguité et ce désaccord disparaissent si l'on suppose que les équations du régime seraient elles- mêmes approximatives et pouvaient être exprimées comme suit :

(équation (IV)): $R=K Q^{0.317} / d^{1 / 6}$ au lieu de $R=K Q^{0,33} / d^{1 / 6}$ (équation (II))

(équation (V)) : $P=K^{\prime} Q^{0,484}$ au lieu de $P=K^{\prime} Q^{0,5}$ (équation (I))

ou bien encore, si l'on admet que le coefficient de rugosité sur fond plat, $k_{r}$, s'exprimait par une relation plus complexe que celle prise en compte et faisait intervenir un paramètre complémentaire, l'allongement $\beta$ par exemple.

Il est probable que la réalité se situe entre ces deux hypothèses. Notons simplement que si nous supposons que l'expression $E$ est indépendante de $Q$ : $E=d^{0.151} / S^{0,19}$, sa valeur calculée pour la Rivière des Galets $(S=2 \%, d=0,21 \mathrm{~m})$ est :

$$
E=1,66
$$

et calculée pour la Jamuna $\left(610^{-5}<S<710^{-5}\right.$, $\left.d=0,1810^{-3} \mathrm{~m}\right)$ :

$$
1,67<E<1,72 .
$$

En moyenne $E=1,7$, soit $S=6,110^{-2} d^{0,79}$.

Nota: Dans les deux exemples ci-dessus, les berges n'ont pratiquement aucune influence sur la rugosité globale du lit. Lorsqu'il n'en est pas ainsi c'est-à-dire si d'une part l'allongement est faible et si d'autre part les talus sont très rugueux (irrégularités, végétaux...), c'est évidemment cette rugosité qui devient prépondérante par rapport à celle du sédiment de fond.

Si $k$ est le coefficient de rugosité globale, (non explicité par rapport à $d$ ), la compatibilité des équations théoriques de l'écoulement avec celles du "régime " conduit alors à la relation suivante :

$$
S \# 40 d^{0.55} / k^{2} Q^{0.1}
$$

$k$ étant une fonction décroissante de $Q$ (la rugosité augmente avec le périmètre de berge intéressé par l'écoulement) la pente $S$ devrait être relativement indépendante du débit.

\section{A propos du paramètre $\beta_{0}$}

Les valeurs $\beta_{0}$ déterminées pour les cas extrêmes que constituent la Jamuna et la Rivière des galets et pour des débits maxima les plus fréquents (limite de débordement), sont de l'ordre de 50 ; autrement dit, pour la plupart des débits dans ces deux cours d'eau dans les conditions du régime :

$$
\beta_{0}<50 .
$$

On peut montrer aisément que de telles valeurs de $\beta_{0}$ correspondent à des valeurs maximales du nombre de 
Froude de l'écoulement et à une dépense minimale d'énergie pour creuser le lit (réf. $a$ et $b$ ); on rejoint là une remarque faite au chapitre A.3.3.5 concernant les phénomènes naturels et qui, dans le domaine fluvial a donné naissance à l'hypothèse du «Minimum stream power " (réf. $k$ ).
Une exception notable est celle que constitue la configuration limite du méandre qui ne pouvant indéfiniment diminuer sa pente se court circuite à son "corps défendant " pour donner naissance au lit en tresses dont l'allongement $\beta$ atteint des valeurs très grandes: de l'ordre de 700 à 800 pour la Jamuna.

\section{Références bibliographiques}

a) Ramette M. - Guide d'hydraulique fluviale. Avril 1981. Rapport HE/40/81/04 du Laboratoire National d'Hydraulique de Chatou (L.N.H.).

b) Ramette M. - Quelques réflexions sur les lois de la morphologie fluviale. 1984. Rapport E40/83/03 du Laboratoire National d'Hydraulique de Chatou (L.N.H.).

c) MARTIN et JAEGGi N. R. - Design of river channels with regard to alternative bars. Congrès IAHR Belgrad. Mai 1980.

d) ENGelund-HANSEN. - A monograph on sediment transport in alluvium streams. T.V. of Denmark. Janvier 1967.

e) Chollet et Cunge. - Simulation of unsteady flow in alluvium streams. Appl. Math. Modelling. Vol. 4. Août 1980.

f) Klassen G. J. et Vermeer K. - Channel characteristics for the braiding Jamuna River. International Conference on River Regime. Wallingford. May 1988.

g) Klassen G. J., Vermeer K. et Nazim Uddin. - Sedimentological processes in the Jamuna (Lower Brahmaputra) river, Bangladesh. International Conference on fluvial hydraulics. Budapest 1988.

h) MPO/Harza. - Total suspended sediment and sand fraction discharge rating curves. Brahmaputra river at Bahadurabad (Bangladesh). July 1985.

i) French Engineering Consortium. - Bangladesh Water Development Board. Prefeasibility study for flood control in Bangladesh. Appendix A 4.14. Vol. 4. Chapter 1. Sand transport problems and river morphology. May 1989.

j) RAMETtE M. - Morphologie et transport solide de rivières à forte pente. La Houille Blanche $n^{\circ} 3 / 4.1988$.

k) CHANG. - Minimum stream power and river channel patterns. Journal of Hydrology $41-303,327-1979$. 